\title{
O MOVIMENTO DOS OLHOS NA DETECÇÃO DE ERROS EM TEXTOS: LEITURA E REVISÃO
}

\author{
Délia Ribeiro Leite ${ }^{1}$ \\ José Olímpio de Magalhães ${ }^{2}$
}

\section{RESUMO}

Este artigo investiga a relação entre leitura e revisão de textos, especificamente quanto à detecção de erros, um dos subprocessos da revisão. Uma tarefa de detecção de erros foi realizada por revisores profissionais e por sujeitos que não trabalham profissionalmente com revisão, utilizando um rastreamento ocular. Primeiramente, verificou-se a proficiência na realização da tarefa. Depois, os padrões das fixações durante a detecção de erros foram relacionados com a proficiência em revisão de textos e com os tipos de erros. Para a detecção de erros de superfície, os sujeitos fizeram uma leitura com um ritmo mais lento, com fixações em média maiores. Já para a detecção de erros mais globais, houve o aumento do número de fixações e do tempo total de leitura, ou seja, os textos foram lidos em um ritmo não tão lento, mas por diversas vezes.

Palavras-chave: Detecção de erros. Revisão de textos. Leitura. Movimento ocular.

\section{INTRODUÇÃO}

A detecção de erros é uma das etapas da revisão de textos. A revisão está diretamente relacionada com o processo de leitura e produção de textos e, por isso, ela é apresentada, nos estudos com abordagem cognitiva, como um subprocesso do processo de escrita, visando melhorar o texto e controlar a produção escrita. No entanto, a revisão pode ocorrer durante o processo de escrita ou separada dele, caso em que é realizada por outra pessoa que não o produtor do texto.

Durante a revisão de textos, podem ocorrer erros de dois tipos (PINTO, 1993): os de composição e os do próprio revisor. Os erros de composição englobam, em 
geral, a não detecção de erros da superfície textual, tais como omissões de letras, palavras ou frases (RIBEIRO, 2007, p. 7). Já os erros do revisor abarcam as falhas relativas ao desconhecimento da língua e são tratados "como "cochilo", o que torna a tarefa de revisar algo para se fazer atenta e detalhadamente" (RIBEIRO, 2007, p. 8). Muitos fatores podem estar envolvidos e trazer como consequência o erro. Um deles pode estar relacionado aos aspectos fisiológicos envolvidos na leitura, como o movimento dos olhos, que é realizado em fixações e sacadas. Outro fator importante é a dificuldade de processamento, pois erros de superfície, como os de ortografia, são quase automaticamente detectados e demandam pouco esforço cognitivo. Já revisões mais globais, como a detecção de problemas de coerência, demandam um esforço cognitivo maior (PIOLAT et al., 2004). Por isso, a leitura também é um componente importante da revisão.

Dessa forma, este trabalho propõe-se a investigar a relação entre leitura e revisão de textos, especificamente quanto à detecção de erros, um dos subprocessos da revisão. Foi utilizado um método de investigação "on line", o rastreamento ocular. Uma tarefa de detecção de erros foi realizada por revisores profissionais e por sujeitos que não trabalham profissionalmente com revisão. Primeiramente, foi verificada a proficiência na realização da tarefa. O objetivo é verificar os padrões das fixações durante a detecção de erros, correlacionando-os com a proficiência em revisão de textos e os tipos de erros.

$\mathrm{Na}$ sessão seguinte, será realizado um levantamento bibliográfico dos estudos sobre revisão, detecção de erros e leitura, sob uma abordagem Psicolinguística. Posteriormente, serão apresentados os métodos da pesquisa e, por fim, os resultados obtidos, bem como os apontamentos que eles suscitam.

\section{REVISÃO DE TEXTOS E LEITURA}

Hayes et al. (1987) elencam as diferenças entre os revisores experientes e os novatos. Os autores destacam que os revisores experientes são mais proficientes para detectar erros do que os novatos, pois os revisores novatos persistem em não detectar erros que são facilmente encontrados pelos experientes. Por meio de uma tarefa de revisão, seguindo a metodologia de protocolos verbais, eles verificaram que os revisores experientes diferem dos novatos quanto à definição da tarefa de revisão, porque têm um maior conhecimento sobre como fazer planos a serem 
seguidos na execução da tarefa, além de criarem antecipadamente um inventário de "problemas" a serem identificados e incluírem objetivos globais de revisão que levam em conta a situação de comunicação. Além disso, os autores verificaram que os revisores experientes frequentemente leem o texto primeiramente para captar sua ideia principal, sem procederem a correções de erros de superfície, que são realizadas em uma segunda leitura. Essa estratégia, porém, não é muito adotada pelos novatos.

Piolat et al. (2004) investigam os tipos de erros e o custo para a memória de trabalho. Dentre os tipos de erros investigados, os mais identificados foram os de ortografia, seguidos dos de sintaxe e, por fim, dos de coerência. Além disso, uma só leitura do texto é suficiente para revisar a maioria dos erros de ortografia; uma ou duas leituras são necessárias para revisar com menos eficácia os erros de sintaxe e ao menos duas leituras são necessárias para revisar somente um terço dos erros de coerência. Por fim, a complexidade da revisão não depende do número de erros a serem corrigidos, mas sim dos tipos de erros. Não houve diferenças na performance dos sujeitos com alta ou baixa memória de trabalho quanto à detecção de erros sintáticos e de coerência, mas houve diferença para os erros de ortografia, porque os com baixa memória de trabalho detectaram menos esses erros.

Já Dedeyan, Largy e Negro (2006) investigam especificamente a relação entre o componente visual e a detecção de erros de concordância verbal, comparando sujeitos de diferentes faixas etárias. Eles concluem que o esboço visuoespacial da memória de trabalho, em especial o seu componente visual, está muito envolvido na revisão experiente.

Portanto, nos estudos que investigam a relação entre revisão e memória de trabalho, a perspectiva geral é a de que os problemas mais globais, de níveis mais altos, geram demandas maiores ao processamento, ao contrário dos problemas de níveis mais baixos, como erros ortográficos e tipográficos, os quais estariam mais relacionados com o componente visual da memória de trabalho. Essas diferenças se espelhariam, ainda, na proficiência em revisão, pois revisores experientes seriam mais proficientes para identificar problemas mais globais, ao contrário dos inexperientes, que se ateriam mais a aspectos da superfície textual.

De qualquer forma, para que a revisão seja realizada, é necessário que se faça a leitura dos textos. A leitura é um processo complexo que, no entanto, é realizado de maneira muito rápida pelo cérebro. 
Just e Carpenter (1980) buscaram estabelecer uma relação direta entre o movimento dos olhos e os aspectos cognitivos envolvidos na leitura. Os autores embasam sua proposta em dois princípios básicos: o princípio de que 0 processamento é incremental e o da ligação olho-mente. Isso significa que o leitor interpreta cada palavra do texto quando a encontra, mesmo que isso the custe fazer suposições que sejam erradas; e os olhos se mantêm focados na palavra o tempo necessário para que ela seja processada.

Os olhos se movimentam em sacadas intercaladas com fixações. Enquanto a sacada corresponde a um rápido movimento dos olhos para mover o foco de uma área para outra, a fixação equivale ao tempo gasto focalizando-se uma determinada área. Em leitura, as fixações duram, em geral, de 120 a 1000 ms; tipicamente, de 200 a 600 ms (RAYNER, 1998, p. 373). No entanto, muitas variáveis podem influenciar esses valores.

Além disso, nem sempre o movimento ocular ocorre de maneira linear, pois há situações em que são realizadas regressões para locais que já foram focalizados. Hyona e Nurminem (2006) investigam as regressões e, em seus resultados, os leitores que tendem a direcionar as regressões para partes realmente informativas do texto conseguem apresentar um resumo mais acurado daquilo que leram.

Já Vauras, Hyona e Niemi (1992) investigam a leitura de textos coerentes e incoerentes. Elas concluem que incoerências aumentam o tempo de fixação e as regressões. No entanto, embora em textos coerentes o aumento do tempo de fixação ocasione melhora na reescrita da parte fixada, o mesmo não ocorre com textos incoerentes.

Alamargot et al. (2006) registram, concomitantemente, o movimento ocular e a execução grafomotora, para investigar o processo de escrita. Eles observam que a detecção de erros tipográficos pode ocorrer em tempos muito curtos, como o de uma fixação na leitura. Pode, ainda, ocorrer em paralelo com a execução grafomotora.

\section{MÉTODOS}

\subsection{Sujeitos}

Dos sujeitos participantes da pesquisa, 14 são universitários que não trabalham profissionalmente com revisão de textos e 14 são profissionais revisores 
de textos, formados em Letras $^{3}$, que trabalham em órgãos públicos, na iniciativa privada ou como autônomos. Dos revisores, à época da pesquisa, 5 trabalhavam com revisão de 1 a 4 anos; 5 , de 5 a 9 anos; e 4, por mais de 10 anos.

Para a seleção dos universitários, era restrição que não cursassem nem Letras nem Comunicação Social, por se considerar que a formação nesses dois cursos é pré-requisito para a investidura em vários cargos de revisor de textos.

Com a seleção de revisores profissionais e de universitários, considera-se que os participantes da pesquisa são leitores proficientes, sendo que um grupo seria mais proficiente em revisão de textos e outro, menos proficiente em revisão.

\subsection{Estímulos}

Os textos, do gênero jornalístico, consistem de um parágrafo. Os problemas dos textos experimentais são de dois tipos: supressão de preposição e incoerência gerada por uma anáfora nominal incorreta. Tais tipos de problemas foram selecionados em função do custo do processamento: enquanto a supressão de preposição se relaciona a níveis mais baixos de processamento, a anáfora incorreta relaciona-se a níveis mais altos. No Anexo $A$, é apresentado um exemplo de texto para cada tipo de erro.

Além disso, foram inseridos textos sem erros e com outros tipos de erros, considerados distratores, os quais tinham de 0 a 4 erros, dentre eles de concordância, ortografia, acentuação, incoerência, digitação e pontuação.

No total, eram 50 textos: 30 distratores e 20 experimentais. Dos experimentais, 10 tinham supressão de preposição e 10, incoerência gerada por uma anáfora nominal incorreta.

Foram controladas variáveis que poderiam influenciar nos resultados: tema; número de sentenças: 3; número de palavras: 54 por texto, 18 por sentença; número de sílabas das palavras alvo: na supressão de preposição, a palavra anterior à preposição tem 3 sílabas e a posterior, 2, e na incoerência tanto o referente quanto a anáfora têm 4 sílabas; posição: nos textos experimentais com supressão de preposição, as palavras alvo são a $15^{\text {a }}$ e $16^{\text {a }}$ do texto e, nos textos com incoerências, a anáfora é a $38^{\mathrm{a}}$ palavra e o referente é a $18^{\mathrm{a}}$ palavra do texto. Além disso, a anáfora e o referente fazem parte de um mesmo campo semântico. 
Como a frequência está bastante relacionada ao tempo de fixação, foi utilizado o corpus Banco de Português (BP), compilado por Tony Sardinha da PUC$\mathrm{SP}$, para se controlar essa variável, sendo que, para as duas condições, foram utilizadas palavras frequentes. Por fim, foi controlado se a palavra alvo era previsível, por meio de um teste de completação. Para os erros de supressão de preposição, a palavra posterior à preposição era previsível, enquanto para a incoerência, as palavras alvo (anáfora e referente) não eram previsíveis.

\subsection{Aplicação do experimento}

O experimento foi realizado no Laboratório de Psicolinguística da Faculdade de Letras da UFMG, utilizando-se o programa EyeLink 1000, da SR Research. Foi solicitado que os participantes lessem os textos, apresentados aleatorizados, procurando identificar erros/inadequações e, ao identificarem, deveriam clicar com o mouse sobre eles.

\section{RESULTADOS}

\subsection{Proficiência na detecção de erros}

Embora a experiência em revisão de textos possa estar correlacionada com a proficiência na detecção de erros, criamos uma árvore de decisão usando o algoritmo CART (Classification and regression trees) (BAAYEN, 2008), para nos certificarmos de que os revisores e não revisores formam grupos que se comportam diferentemente quanto à marcação dos erros.

Para essa análise, foi utilizada como variável dependente a marcação ou não dos erros, tendo como base tanto os textos experimentais quanto os distratores. Dessa forma, cada sujeito deveria identificar 81 erros, além de lerem 6 textos em que não havia erros.

A variável dependente, marcação esperada para o erro, é, portanto, uma binomial (marcou ou não marcou). Foram variáveis independentes: o sujeito, o tipo de erro, o número de erros de cada sentença e a experiência em revisão. 
A árvore mais adequada, tendo em vista a relação entre economia dos preditores e validade do modelo ${ }^{4}$, é apresentada na Figura 1 , e sua descrição no Quadro 1.

FIGURA 1: ÁRVORE DE DECISÃO

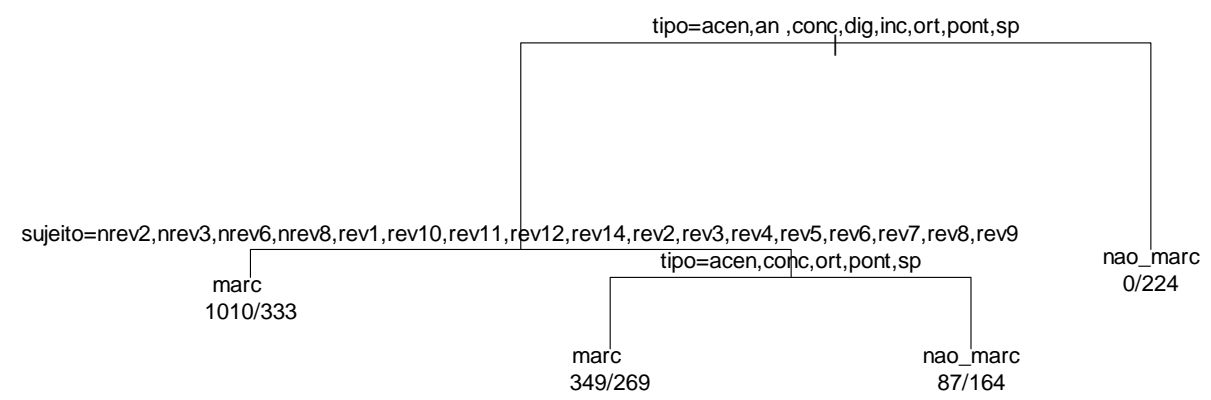

$n=2436$

1) root: em 2436, marcou $=1446(p=0,594)$ e não marcou $=990(p=0,406)$

2) tipo= acen (acentuação), an (anáfora), conc (concordância), dig (digitação), inc (incoerência), ort (ortografia), pont (pontuação) e sp (supressão de preposição): marcou $=2212$ e não marcou $=766(0,654$ e 0,346)

4) sujeito= nrev2,nrev3,nrev6,nrev8,rev1,rev10,rev11,rev12,rev14,rev2,rev3, rev4, rev5, rev6, rev7,rev8, rev9: marcou $=1343(p=0,752)$ e não marcou $333(p=0,248)$ *

5) sujeito= nrev1,nrev10,nrev11,nrev12,nrev13,nrev14,nrev4,nrev5,nrev7, nrev9,rev13: marcou $=869(p=0,501)$ e não marcou $=433(p=0,498)$

10) tipo=acen,conc,ort,pont,sp: marcou $=618(p=0,565)$ e não marcou $=$ $269(p=0,435)$ *

11) tipo=an, dig,inc: não marcou=251 $(p=0,653)$ e marcou $=87(p=0.347)$ *

3) tipo= sem erro: em 224, não marcou $=224(p=1)$ *

Emergem como preditores o tipo de erro e os sujeitos. Quanto ao tipo de erro, há diferença entre quando há erro, com a probabilidade geral de marcação de 0,65, e entre quando não há erro, com a probabilidade de 0 . Os sujeitos são classificados 
em dois grupos: um mais proficiente, composto por 13 revisores e 4 não revisores, cuja probabilidade de marcação dos erros é de 0,75; outro com proficiência menor, composto por 11 sujeitos, 10 não revisores e 1 revisor $^{5}$, cuja probabilidade de marcação dos erros é de 0,50 , sendo que, quando o erro é de anáfora incorreta, digitação ou incoerência, a probabilidade deste último grupo cai para 0,34.

Dessa forma, os dados, para as análises seguintes, foram agrupados conforme a classificação que emergiu:

- Grupo OP: representa a não proficiência, composto de todos os sujeitos, quando eles não marcaram o erro;

- Grupo +P: representa maior proficiência, composto de 17 sujeitos;

- Grupo -P: representa menor proficiência, composto de 11 sujeitos.

\subsection{Tempo total de leitura dos textos}

Essa variável pode indicar uma dificuldade no processamento do texto e, assim, dos erros. Os resultados descritivos são compilados na Tabela 1.

\begin{tabular}{l|l|l|l|l|l|l}
\hline \multicolumn{7}{c}{ Tabela 1 - Tempo total de leitura } \\
\hline \multirow{2}{*}{ Grupo } & Condição & $\mathbf{N}^{6}$ & Mediana(ms) & Média(ms) & $\begin{array}{l}\text { Desvio- } \\
\text { Padrão }\end{array}$ & $\begin{array}{l}\text { p-valor } \\
\text { Shapiro- } \\
\text { Wilk }\end{array}$ \\
\hline \multirow{2}{*}{ OP } & Preposição & 37 & 29020 & 31270 & 11830 & 0,004 \\
\cline { 2 - 8 } & Anáfora & 167 & 28510 & 30870 & 12185 & 0,000 \\
\hline \multirow{2}{*}{$+\mathrm{P}$} & Preposição & 148 & 27780 & 31320 & 11674 & 0,000 \\
\cline { 2 - 7 } & Anáfora & 77 & 35410 & 37170 & 12863 & 0,003 \\
\hline \multirow{2}{*}{$-\mathrm{P}$} & Preposição & 94 & 25170 & 27100 & 9369 & 0,008 \\
\cline { 2 - 7 } & Anáfora & 36 & 28010 & 28710 & 8996 & 0,168 \\
\hline
\end{tabular}

Como foram violados os pressupostos do teste estatístico paramétrico ANOVA, já que as distribuições não são normais e as variâncias não são iguais, foi aplicado o teste correspondente não paramétrico, Kruskal-Wallis, no qual obtivemos um p-valor $(0,000)$ que nos permite, assumindo um nível de significância de $95 \%$, rejeitar a hipótese nula e assumir a alternativa de que, nessa amostra, os grupos não provêm de uma mesma população. Realizamos, então, testes cruzados utilizando o teste não paramétrico Mann-Whitney, com a correção de Bonferroni. Os resultados são apresentados abaixo: 


\begin{tabular}{|c|c|c|c|c|}
\hline \multicolumn{5}{|c|}{ Tabela 2 - Testes Mann-Whitney - tempo total de leitura } \\
\hline Condição & Grupo & Condição & Grupo & p-valor \\
\hline \multirow[t]{3}{*}{ Preposição } & $0 p$ & \multirow[t]{3}{*}{ Preposição } & $+\mathrm{P}$ & 1 \\
\hline & OP & & $-\mathrm{P}$ & 1 \\
\hline & $+\mathrm{P}$ & & $-P$ & 0,224 \\
\hline \multirow[t]{3}{*}{ Anáfora } & OP & \multirow[t]{3}{*}{ Anáfora } & $+\mathrm{P}$ & $0,003^{* \star}$ \\
\hline & OP & & $-\mathrm{P}$ & 1 \\
\hline & $+\mathrm{P}$ & & $-\mathrm{P}$ & 0,01 * \\
\hline \multirow[t]{9}{*}{ Preposição } & $0 p$ & \multirow[t]{9}{*}{ Anáfora } & $+P$ & 0,09 . \\
\hline & OP & & $-P$ & 1 \\
\hline & $+\mathrm{P}$ & & OP & 1 \\
\hline & $-P$ & & OP & 0,611 \\
\hline & $+P$ & & $-P$ & 1 \\
\hline & $-P$ & & $+P$ & $0,000^{* \star \star}$ \\
\hline & $+\mathrm{P}$ & & $+P$ & $0,005^{* *}$ \\
\hline & $-\mathrm{P}$ & & $-\mathrm{P}$ & 1 \\
\hline & OP & & OP & 1 \\
\hline
\end{tabular}

Os resultados mais relevantes para este parâmetro são relativos à incoerência gerada pela anáfora incorreta. Isso porque, nesse caso, há diferença estatisticamente significativa entre o grupo mais proficiente $(+\mathrm{P})$ e os grupos não proficiente $(\mathrm{OP})$ e menos proficiente $(-\mathrm{P})$, sendo que o primeiro grupo $(+\mathrm{P})$ teve, em geral, valores mais elevados de tempo total de leitura.

Isso indica que o aumento do tempo de leitura do texto ocasiona o aumento da probabilidade de se detectar esse tipo de erro e, assim, a proficiência. Neste caso, especificamente, há uma separação entre o grupo $+\mathrm{P}$ e os grupos $-\mathrm{P}$ e $0 \mathrm{P}$. Esses dois últimos grupos, portanto, são semelhantes no que se refere ao tempo total de leitura, o que indica que um menor tempo total de leitura não acarreta necessariamente que o erro não seja identificado, mas sim diminui as chances de que o erro seja identificado e, também, a proficiência.

Além disso, o grupo mais proficiente $(+\mathrm{P})$ diferiu quando marcava a anáfora e a supressão de preposição, o que pode indicar uma diferença na dificuldade de processamento desses dois tipos de erros para este grupo, tendo sido mais custosos (maior tempo) os textos com a incoerência, o que não ocorreu com os grupos OP e -P. 


\subsection{Número de fixações no texto}

A Tabela 3 sumariza os resultados referentes ao número de fixações nos textos.

\begin{tabular}{l|l|l|l|l|l}
\hline \multicolumn{7}{c}{ Tabela 3 - número de fixações nos textos } \\
\hline \multirow{2}{*}{ Grupo } & Condição & $\mathbf{n}$ & Mediana(ms) & Média(ms) & $\begin{array}{l}\text { Desvio- } \\
\text { Padrão }\end{array}$ \\
\hline \multirow{2}{*}{ OP } & Preposição & 37 & 60 & 256 & 113 \\
\cline { 2 - 7 } & Anáfora & 167 & 49 & 262 & 112 \\
\hline$+\mathrm{P}$ & Preposição & 148 & 66 & 264 & 104 \\
\cline { 2 - 7 } & Anáfora & 77 & 68 & 283 & 133,5 \\
\hline \multirow{2}{*}{$-\mathrm{P}$} & Preposição & 94 & 49 & 235 & 103 \\
\cline { 2 - 6 } & Anáfora & 36 & 49 & 235 & 103 \\
\hline
\end{tabular}

Como a variável dependente é ordinal, realizamos uma regressão logística ordinal, tendo como variável dependente o número de fixações nos textos e, como variáveis preditoras, as condições e os grupos.

Os resultados obtidos são apresentados no Quadro 2.

\begin{tabular}{|c|c|c|c|c|}
\hline \multicolumn{5}{|c|}{$\begin{array}{l}\text { QUADRO 2: REGRESSÃO LOGÍSTICA ORDINAL - NÚMEROS DE FIXAÇÕES } \\
\text { NOS TEXTOS }\end{array}$} \\
\hline NULL & $\begin{array}{l}\text { Deviance } \\
838\end{array}$ & $\begin{array}{l}\text { Resid. Df } \\
1775260\end{array}$ & Resid. Dev & $\mathrm{P}(>\mid$ Chi $\mid)$ \\
\hline grupos & 3573 & 837 & 1771687 & 0.187221 \\
\hline condição 1 & 41394 & 836 & 1730293 & $7.153 \mathrm{e}-06^{* * *}$ \\
\hline grupo:cond 1 & 15111 & 835 & 1715182 & $0.006682^{* *}$ \\
\hline
\end{tabular}

Houve diferença estatisticamente significativa entre os grupos e na interação entre os grupos e as condições. Por isso, aplicamos o teste Mann-Withney com a correção de Bonferroni. 


\begin{tabular}{|c|c|c|c|c|}
\hline \multicolumn{5}{|c|}{ TABELA 4: TESTES MANN-WHITNEY - NÚMERO DE FIXAÇÕES } \\
\hline Condição & Grupo & Condição & Grupo & p-valor \\
\hline \multirow[t]{3}{*}{ Preposição } & $\mathrm{OP}$ & \multirow[t]{3}{*}{ Preposição } & $+\mathrm{P}$ & 0,832 \\
\hline & OP & & $-\mathrm{P}$ & 0,435 \\
\hline & $+\mathrm{P}$ & & $-\mathrm{P}$ & 1 \\
\hline \multirow[t]{3}{*}{ Anáfora } & OP & \multirow[t]{3}{*}{ Anáfora } & $+\mathrm{P}$ & $0,009 * *$ \\
\hline & OP & & $-\mathrm{P}$ & 1 \\
\hline & $+\mathrm{P}$ & & $-\mathrm{P}$ & 0,187 \\
\hline \multirow[t]{9}{*}{ Preposição } & OP & \multirow[t]{9}{*}{ Anáfora } & $+\mathrm{P}$ & 0,435 \\
\hline & OP & & $-P$ & 1 \\
\hline & $+\mathrm{P}$ & & OP & 1 \\
\hline & $-\mathrm{P}$ & & OP & 1 \\
\hline & $+\mathrm{P}$ & & $-P$ & 1 \\
\hline & $-P$ & & $+P$ & $0,000^{* * *}$ \\
\hline & $+\mathrm{P}$ & & $+P$ & $0,000^{* * *}$ \\
\hline & $-P$ & & $-\mathrm{P}$ & 1 \\
\hline & OP & & OP & 1 \\
\hline
\end{tabular}

Para esse parâmetro, foi estatisticamente significativa a diferença entre os grupos +P e OP, para a condição de anáfora incorreta. Isso indica uma tendência de, ao se aumentar o número de fixações nos textos, aumentar também a probabilidade de se identificar esse tipo de erro e, assim, favorecer a proficiência. No entanto, como não houve diferença estatisticamente significativa entre os grupos $-\mathrm{P} e+P$, assim como entre $\mathrm{OP}$ e -P, só é possível identificar essa tendência. De qualquer forma, o grupo $+\mathrm{P}$ na condição de anáfora tem um padrão bastante diferente, tanto que também há diferença estatisticamente significativa para este grupo e $-P$ na condição de supressão de preposição, bem como, no próprio grupo $+\mathrm{P}$, entre as condições de supressão de preposição e de anáfora incorreta.

\subsection{Duração média das fixações no texto}

Os resultados descritivos são apresentados na Tabela 5. 


\begin{tabular}{l|l|l|l|l|l|l}
\hline \multicolumn{7}{c}{ Tabela 5 - duração média das fixações } \\
\hline \multirow{2}{*}{ Grupo } & Condição & $\mathbf{n}$ & Mediana(ms) & Média(ms) & $\begin{array}{l}\text { Desvio- } \\
\text { Padrão }\end{array}$ & $\begin{array}{l}\text { p-valor } \\
\text { Shapiro- } \\
\text { Wilk }\end{array}$ \\
\hline \multirow{2}{*}{ OP } & Preposição & 37 & 197,8 & 203,9 & 24,7 & 0,003 \\
\cline { 2 - 8 } & Anáfora & 167 & 206,5 & 212,3 & 26,11 & 0,000 \\
\hline \multirow{2}{*}{$+\mathrm{P}$} & Preposição & 148 & 220,8 & 225,8 & 33,9 & 0,050 \\
\cline { 2 - 8 } & Anáfora & 77 & 214,2 & 217,3 & 36,0 & 0,251 \\
\hline \multirow{2}{*}{$-\mathrm{P}$} & Preposição & 94 & 209,2 & 211,6 & 19,8 & 0,001 \\
\cline { 2 - 7 } & Anáfora & 36 & 196,5 & 200,9 & 15,4 & 0,080 \\
\hline
\end{tabular}

Como o p valor do teste Kruskal-Wallis foi 0,000, realizamos testes MannWhitney com correção de Bonferroni, cujos resultados são apresentados na Tabela 6.

\begin{tabular}{|c|c|c|c|c|}
\hline \multicolumn{5}{|c|}{ Tabela 6 - testes Mann-Whitney - duração média das fixações } \\
\hline Condição & Grupo & Condição & Grupo & p-valor \\
\hline \multirow[t]{3}{*}{ Preposição } & OP & \multirow[t]{3}{*}{ Preposição } & $+\mathrm{P}$ & $0,000^{* * \star}$ \\
\hline & OP & & $-\mathrm{P}$ & 0,135 \\
\hline & $+\mathrm{P}$ & & $-P$ & $0,008^{* *}$ \\
\hline \multirow[t]{3}{*}{ Anáfora } & OP & \multirow[t]{3}{*}{ Anáfora } & $+\mathrm{P}$ & 1 \\
\hline & OP & & $-\mathrm{P}$ & 0,372 \\
\hline & $+\mathrm{P}$ & & $-P$ & 0,085 * \\
\hline \multirow[t]{9}{*}{ Preposição } & OP & \multirow[t]{9}{*}{ Anáfora } & $+\mathrm{P}$ & 0,105 \\
\hline & OP & & $-\mathrm{P}$ & 1 \\
\hline & $+\mathrm{P}$ & & OP & $0,001^{* *}$ \\
\hline & $-P$ & & OP & 1 \\
\hline & $+\mathrm{P}$ & & $-\mathrm{P}$ & $0,000^{* \star \star}$ \\
\hline & $-P$ & & $+\mathrm{P}$ & 1 \\
\hline & $+\mathrm{P}$ & & $+P$ & 1 \\
\hline & $-P$ & & $-P$ & 0,064 \\
\hline & OP & & OP & 0,330 \\
\hline
\end{tabular}

$\mathrm{Na}$ supressão de preposição, houve diferença entre o grupo $+\mathrm{P}$ e os grupos OP e -P. Portanto, com relação à duração média das fixações nos textos, os grupos $-P$ e $O P$ não diferiram entre si, mas, ao contrário, foram diferentes do grupo $+P$, sendo que este apresentou, em geral, valores mais elevados para este parâmetro.

Portanto, o aumento da duração média das fixações nos textos está correlacionado com o aumento da probabilidade de se identificar o erro de supressão de preposição, assim como com a proficiência na detecção desse tipo de erro. Nota-se que a duração média das fixações do grupo $+P$ para a condição de 
supressão de preposição é bastante diferenciada, tanto que este grupo difere-se também do $\mathrm{OP}$ e do $-\mathrm{P}$ na condição de anáfora incorreta.

\subsection{Tempo total de fixação na palavra alvo}

Quanto a esse parâmetro, os resultados descritivos são apresentados na Tabela 7.

\begin{tabular}{l|l|l|l|l|l|l}
\hline \multicolumn{7}{c}{ Tabela 7 - tempo total de fixação na palavra alvo } \\
\hline Grupo & Condição & $\mathbf{n}$ & Mediana(ms) & Média(ms) & $\begin{array}{l}\text { Desvio- } \\
\text { Padrão }\end{array}$ & $\begin{array}{l}\text { p-valor } \\
\text { Shapiro- } \\
\text { Wilk }\end{array}$ \\
\hline \multirow{2}{*}{ OP } & Preposição & 37 & 1070 & 1652 & 1725 & 0,004 \\
\cline { 2 - 8 } & Anáfora & 334 & 521,5 & 748,6 & 602,6 & 0,000 \\
\hline \multirow{2}{*}{$+\mathrm{P}$} & Preposição & 148 & 2942 & 3279 & 1539 & 0,000 \\
\cline { 2 - 7 } & Anáfora & 154 & 1884 & 2045 & 1082 & 0,000 \\
\hline \multirow{2}{*}{$-\mathrm{P}$} & Preposição & 94 & 3595 & 3923 & 2003 & 0,000 \\
\cline { 2 - 7 } & Anáfora & 72 & 1938 & 2026 & 703,9 & 0,610 \\
\hline
\end{tabular}

Como o teste Kruskal-Wallis foi significativo $(0,000)$, foram aplicados os testes Mann-Whitney com a correção de Bonferroni, cujos resultados são apresentados na Tabela 8.

\begin{tabular}{|c|c|c|c|c|}
\hline \multicolumn{5}{|c|}{ Tabela 8 - testes Mann-Whitney - tempo total de fixação } \\
\hline Condição & Grupo & Condição & Grupo & p-valor \\
\hline \multirow[t]{3}{*}{ Preposição } & OP & \multirow[t]{3}{*}{ Preposição } & $+\mathrm{P}$ & $0,000^{* * *}$ \\
\hline & OP & & $-P$ & $0,000 * * *$ \\
\hline & $+\mathrm{P}$ & & $-P$ & 0,207 \\
\hline \multirow[t]{3}{*}{ Anáfora } & $\mathrm{OP}$ & \multirow[t]{3}{*}{ Anáfora } & $+\mathrm{P}$ & $0,000^{* * \star}$ \\
\hline & OP & & $-P$ & $0,000^{* * *}$ \\
\hline & $+\mathrm{P}$ & & $-P$ & 1 \\
\hline \multirow[t]{9}{*}{ Preposição } & OP & \multirow[t]{9}{*}{ Anáfora } & $+\mathrm{P}$ & $0,000^{* * *}$ \\
\hline & $\mathrm{OP}$ & & $-P$ & $0,001^{* *}$ \\
\hline & $+\mathrm{P}$ & & OP & $0,000^{* * \star}$ \\
\hline & $-P$ & & $\mathrm{OP}$ & $0,000^{* * *}$ \\
\hline & $+\mathrm{P}$ & & $-P$ & $0,000^{* * *}$ \\
\hline & $-\mathrm{P}$ & & $+\mathrm{P}$ & $0,000^{* * *}$ \\
\hline & $+\mathrm{P}$ & & $+\mathrm{P}$ & $0,000^{* * \star}$ \\
\hline & $-\mathrm{P}$ & & $-\mathrm{P}$ & $0,000^{* * *}$ \\
\hline & $\mathrm{OP}$ & & OP & $0,000 * * *$ \\
\hline
\end{tabular}

Não houve diferença significativa somente quanto aos grupos $+\mathrm{P}$ e $-\mathrm{P}$ tanto para a condição de anáfora incorreta quanto para a condição de supressão de 
preposição. Portanto, levando em conta o tempo total de fixação na palavra alvo, esses dois grupos não diferiram, mas se contrapõem ao grupo OP.

O tempo total de fixação na palavra alvo, portanto, mostrou-se um parâmetro extremamente significativo para a detecção de ambos os tipos de erros, mas não é determinante para se potencializar a proficiência. Como todos os grupos $(0 \mathrm{P},+\mathrm{P}$ e P) diferiram entre si quando se tratava de condições diferentes, podemos considerar, ainda, que o tempo total de fixação na palavra alvo é determinante no que se refere ao impacto de cada tipo de erro no processamento.

\section{CONSIDERAÇÕES FINAIS}

Com a árvore de decisão, foi possível reclassificar os sujeitos conforme a proficiência na detecção de erros. A classificação que emergiu foi relativamente semelhante à divisão dos grupos conforme a experiência em revisão, já que somente 5 (18\%) dos 28 sujeitos foram reclassificados, 1 revisor $(3,5 \%)$ e 4 não revisores $(14,5 \%)$.

Com a árvore que emergiu, foi possível identificar 3 grupos: um não proficiente (OP), um menos proficiente $(-P)$ e um mais proficiente $(+P)$. Nesses dois últimos grupos, a porcentagem de detecção dos erros de supressão de preposição foi maior do que a dos erros de anáfora incorreta, o que vai de encontro à literatura, segundo a qual erros de níveis mais altos geram mais demandas ao processamento e são mais dificilmente identificados.

Quando houve incoerência gerada por uma anáfora nominal incorreta, o tempo total de leitura do texto foi um parâmetro importante para a detecção do erro e para a proficiência, sendo que o aumento desse tempo tende a estar relacionado com o aumento do número de fixações no texto, e não com o aumento do tempo médio das fixações.

O aumento do tempo médio das fixações, por sua vez, está relacionado com a detecção de erros de supressão de preposição, bem como com a proficiência nesse caso.

Piolat et al. (2004) verificaram que uma só leitura do texto é suficiente para revisar a maioria dos erros de ortografia e ao menos duas leituras são necessárias para revisar somente um terço dos erros de coerência. No entanto, os autores não investigaram quais diferenças ocorrem na forma de ler os textos com cada tipo de 
erro. Os resultados do trabalho ora apresentado indicam que há diferença na maneira de ler que faz com que se aumentem as chances de detectar cada tipo de erro.

Para a detecção de um erro mais global, tal como a incoerência gerada pela anáfora incorreta, o aumento do tempo total de leitura do texto tende a estar relacionado com o aumento do número de fixações nos textos. Portanto, a maior demora na leitura desse tipo de texto é decorrente de mais fixações, possivelmente relacionadas a mais de uma leitura do texto ou a vários retornos à região crítica. Já para a detecção de um erro de superfície, como a supressão de preposição, é importante que as fixações sejam em média maiores, ou seja, que tenhamos uma leitura com ritmo mais lento.

Essa diferença no padrão de leitura relacionada ao processamento e à detecção de cada tipo de erro pode explicar o fato de revisores experientes frequentemente lerem o texto primeiramente para captar sua ideia principal, sem procederem a correções de erros de superfície, que são realizadas em uma segunda leitura (HAYES et al., 1987). Essa estratégia, portanto, pode ser decorrente da relação entre o processamento de cada nível do texto e o padrão de fixações na leitura.

Portanto, o estudo ora apresentado é importante tanto por investigar a relação entre leitura e detecção de erros, quanto por apontar formas de aumentar a proficiência na detecção e, assim, na revisão de textos. Para a detecção de erros de superfície, é indicada uma leitura com um ritmo mais lento, com fixações em média maiores, o que pode diminuir as chances de ocorrer um erro de composição (PINTO, 1993). Já para a detecção de erros mais globais, é indicado aumentar o número de fixações, ou seja, ler o texto em um ritmo não tão lento, mas por diversas vezes.

Por fim, um parâmetro determinante para explicar a deteç̧ão de erros é o tempo total de fixação no trecho crítico, pois, quando um erro é identificado, isso acarreta o aumento do tempo de fixação no trecho que contém o erro. O tempo total de fixação no trecho crítico é ainda diferenciado para cada tipo de erro e, assim, tem uma relação cabal com os níveis de processamento.

\section{NOTAS}

${ }^{1}$ Doutoranda em Linguística pelo Programa de Pós-Graduação em Linguística da Universidade Federal de Minas Gerais. 
${ }^{2}$ Professor titular do Departamento de Letras da Universidade Federal de Minas Gerais.

${ }^{3}$ Somente um dos revisores cursava, à época, o último período de Letras.

${ }^{4}$ Para a escolha da árvore mais adequada, utilizamos o método pós-poda "cost-complexity pruning". Para mais informações, consultar Baayen (2008, p. 160-167).

${ }^{5} \mathrm{O}$ revisor reclassificado não era o que, à época, cursava Letras.

${ }^{6}$ Para a condição de supressão de preposição, $n$ é igual a 279, e não 280 (28 sujeitos vezes 10 textos) porque um texto teve de ser descartado devido a uma falha do equipamento.

\title{
EYE MOVEMENTS ON THE DETECTION OF ERRORS IN TEXTS: READING AND REVISION
}

\begin{abstract}
This paper reports a research on the relation between the reading and the text revision, specially the detection of errors, one subprocess of the text revision. One experiment with detection of errors was done by professional revisers and by subjects that don't work with revision, using an eye tracker. Initially, it was analyzed the proficiency in the realization of the experiment. After, the patterns of fixations in the detection of the errors were related with the proficiency in text revision and with the types of errors. To the detection of the surface errors, the subjects read the texts with a slower rhythm, with generally larger fixations. To the detection of more global errors, there was the increase of the number of fixations, in other words the texts were read with a rhythm not so slow, but repeatedly.
\end{abstract}

Keywords: Detection of errors. Text revision. Reading. Eye movements.

\section{REFERÊNCIAS}

ALAMARGOT, D. et al. Eye and pen: a new device to study reading during writing. Behaviour Research Methods, Instruments and Computers, v. 2, n. 38, p. 287-299, 2006.

BAAYEN, R. H. Analyzing linguistic data: a practical introduction to statistics using R. Cambridge: Cambridge University Press, 2008. 
DEDEYAN, Al.; LARGY, P.; NEGRO, I. Mémoire de travail et détection d'erreurs d'accord verbal: étude chez le novice et l'expert. Langages, n. 164, p. 57-70, 2006.

HAYES J.R. et al. Cognitive processes in revision. In ROSENBERG, S (Ed.). Advances in psycholinguistics: v. II: reading, writing, and language processing. Cambridge: Cambridge University Press, 1987. p. 176-240.

HYONA, J.; NURMINEN, A. M. Do adult readers know how they read? Evidence from eye movement patterns and verbal reports. British Journal of Psychology, n. 97, p. 31-50, 2006.

JUST, M. A.; CARPENTER, P. A. A theory of reading: from eye fixations to comprehension. Psychological Review, v. 87, n. 4, p. 329-354, 1980.

PINTO, Ildete Oliveira. O livro: manual de preparação e revisão. São Paulo: Ática, 1993.

PIOLAT, A. et al. Text revision and working memory. In: ALLAL, L.; CHANQUOY, L.; LARGY, P. Revision: cognitive and instructional processes. EUA: Kluwer Academic Publishers, 2004. p. 21-38.

RAYNER, K. Eye movements in reading and information processing: 20 years of research. Psychological Bulletin, v. 124, n. 3, p. 372-422, 1998.

RIBEIRO, A. E. Em busca do texto perfeito: (in)distinções entre as atividades do editor de texto e do revisor de provas na produção de livros. In: CONGRESSO BRASILEIRO DE CIÊNCIAS DA COMUNICAÇÃOO DA REGIÃO SUDESTE, 9., 2007, Juiz de Fora. Anais... Juiz de Fora: Sociedade Brasileira de Estudos Interdisciplinares da Comunicação, 2007, p. 1-14. Disponível em: $<$ http://www. portcom. intercom.org.br/navegacaoDetalhe.php?option=trabalho\&id=28 804>. Acesso em 11 nov. 2013.

VAURAS, M.; HYONA, J.; NIEMI, P. Comprehending coherent and incoherent texts: evidence from eye movement patterns and recall performance. Journal of Research in Reading, v.15, n. 1, p. 39-54, 1992. 


\begin{abstract}
ANEXO A
Exemplo de texto com supressão de preposição

Com a facilidade de financiamento, a venda de imóveis cresceu em São Paulo de janeiro julho deste ano. O paulista pode realizar o sonho da casa própria e também aproveitar o mercado de imóveis em alta. A expectativa é de que o setor tenha um crescimento maior com a queda dos juros dos bancos.
\end{abstract}

Exemplo de texto com incoerência gerada por uma anáfora nominal incorreta

No Shopping Del Rey, uma pasta com trinta mil reais foi esquecida em uma loja por um deputado. Uma das vendedoras da loja encontrou a pasta e ligou para o número de celular anotado na etiqueta. O governador ficou tão feliz em recuperar todo o dinheiro perdido que até pretende gratificar a honesta vendedora. 